\title{
A WEAR GEOMETRY MODEL OF PLAIN WOVEN FABRIC COMPOSITES
}

\author{
Dapeng Gu ${ }^{1,2 *}$, Yulin Yang ${ }^{1,2}$, Suwen Chen ${ }^{1,2}$, Wenwen Su ${ }^{1,2}$ \\ ${ }^{1}$ College of Mechanical Engineering, Yanshan University, Qinhuangdao, Hebei, China; \\ ${ }^{2}$ Aviation Key Laboratory of Science and Technology on Generic Technology of Self-lubricating Spherical Plain Bearing, \\ Yanshan University, Qinhuangdao, Hebei, China \\ E-mail: jsgudapeng@ysu.edu.cn
}

\begin{abstract}
:
The paper $g$ describes a model meant for analysis of the wear geometry of plain woven fabric composites. The referred model consists of a mathematical description of plain woven fabric based on Peirce's model coupled with a stratified method for the solution of the wear geometry. The evolutions of the wear area ratio of weft yarn, warp yarn and matrix resin on the worn surface are simulated by MatLab software in combination of warp and weft yarn diameters, warp and weft yarn-to-yarn distances, fabric structure phases (SPS). By comparing theoretical and experimental results from the PTFE/Kevlar fabric wear experiment, it can be concluded that the model can present a trend of the component area ratio variations along with the thickness of fabric, but has a inherently large error in quantitative analysis as an idealized model.
\end{abstract}

\section{Keywords:}

Wear geometry, woven fabric composites, stratified method

\section{Introduction}

Woven fabric composites are widely used as frictional materials by virtue of their excellent tribological properties in the automotive, aerospace and aeronautical industries. In general, they are fabricated by hybrid fabrics, in which the materials and the geometric shapes of the warp yarn and weft yarn are different, impregnated with matrix resin. In other words, they are three-component material systems composed of weft yarn, warp yarn and matrix resin. Excellent tribological properties could be obtained by combining the benefit of each component into a single composite product. In tribological studies of such materials, one of the emphases lays on the wear resistance performance [1-3]. Various factors such as type and amount of matrix resin and fabric, fiber-matrix adhesion along with processing technology, working condition control the antiwear performance of fabric composites [4]. Recently, it has been demonstrated that textile structures showed an important impact on the wear performance of such materials [5-7]. Textile structures make the component distribution of such materials uneven, thus the wear resistance changes with the variation of wear geometry caused by the textile structure during the wear process [8]; namely, the wear area ratio of weft yarn, warp yarn and matrix resin on the worn surface changes with the wear depth change. However, literature is scarce on the wear geometry model of woven fabric composites. Hence, in this paper, a wear geometry model of plain woven fabric composites is established. The evolutions of the wear area ratio of weft yarn, warp yarn and matrix resin on the worn surface are simulated by MatLab software in combination of warp and weft yarn diameters, warp and weft yarn-to-yarn distances, fabric SP.

\section{Wear geometry model}

\subsection{Geometrical relationships}

The geometrical description of the textile structure of plain woven fabric is based on the Peirce model [9]. As seen in Figure 1, the cross sections of warp and weft yarns are assumed to be circular, and the flexion shapes of warp and weft yarns are described by arcs and tangent lines. Two basic geometrical parameters $l_{\mathrm{f}}$ and $I_{w}$ denote, respectively, the yarn-to-yarn distance of two adjacent weft yarns and two adjacent warp yarns.

Figure 2 shows the geometric description of half a unit cell of weft yarn. The centre line of weft yarn is composed of one tangent line and two arcs which are concentric with warp yarn circular cross sections. The diameters of weft and warp yarns circular cross sections are, respectively, $d_{f}$ and $d_{w}$. The flexion heights of weft and warp yarns are, respectively, $h_{\mathrm{f}}$ and $h_{\mathrm{w}}$. Thus, the length of tangent line of weft yarn, $p_{f}$, the flexion angle of weft yarn, $\theta_{f}$, the height of weft yarn, $h_{1}$, and the height of warp yarn, $h_{2}$, can be expressed as follows.

$$
\begin{aligned}
& p_{\mathrm{f}}=\sqrt{I_{\mathrm{w}}^{2}+h_{\mathrm{w}}^{2}-\left(d_{\mathrm{w}}+d_{\mathrm{f}}\right)^{2}} \\
& \theta_{\mathrm{f}}=\arcsin \left\{\left[I_{\mathrm{w}}\left(d_{\mathrm{f}}+d_{\mathrm{w}}\right)-h_{\mathrm{w}} p_{\mathrm{f}}\right] /\left(I_{\mathrm{w}}^{2}+h_{\mathrm{w}}^{2}\right)\right\} \\
& h_{1}=h_{\mathrm{f}}+d_{\mathrm{f}}=d_{\mathrm{w}}+2 d_{\mathrm{f}}-h_{\mathrm{w}} \\
& h_{2}=h_{\mathrm{w}}+d_{\mathrm{w}}=d_{\mathrm{f}}+2 d_{\mathrm{w}}-h_{\mathrm{f}}
\end{aligned}
$$

According to Eqs. (3) and (4), the following relationships can be obtained, 


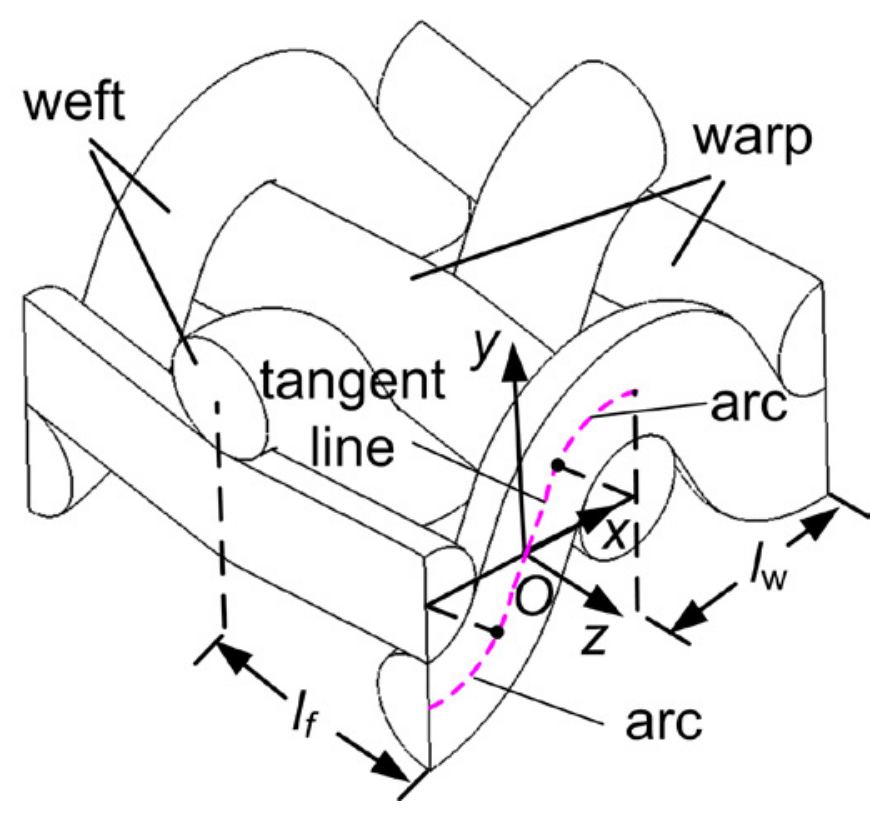

Figure 1. A unit cell of plain woven fabric

$$
h_{\mathrm{f}}+h_{\mathrm{w}}=d_{\mathrm{w}}+d_{\mathrm{f}}, \quad h_{1}+h_{2}=2\left(d_{\mathrm{w}}+d_{\mathrm{f}}\right)
$$

The thickness $h$ of plain woven fabric depends upon $h_{1}$ or $h_{2}$, $h=\max \left(h_{1}, h_{2}\right)$.

The three-dimensional mathematical model of plain woven fabric is established on the basis of the coordinate transformation as described elsewhere [10].

\subsection{Three-dimensional mathematical model}

Based on the geometric characterisation of plain woven fabric, a numerical analysis stratified method, taking weft yarn as an example, is presented to determine the wear geometry function. Considering the symmetry and periodicity with $x= \pm I_{w} / 2$ and $z$ $=0$, this paper only takes a quarter unit cell of weft yarn for the following study, in which the range of the $x$ coordinate is $-I_{w} / 2$ $\leq x \leq 1 / 2$ and of the $z$ coordinate is $z \geq 0$, as shown in Figure 4. According to this method, fabric is divided into $m-1$ layers along the thickness. In Figure (4a), the $m$ - 1 layers of weft yarn are, respectively, described as the 1 st layer, the 2 nd layer, .... the $(m-1)$ th layer, from bottom to top along the positive direction of $y$ axis. The $m-1$ layers have $m$ horizontal sections, where the $y$ coordinates are, respectively, expressed as $y_{1}, y_{2}, \ldots, y m$.

As depicted in Figure $4 b$, selecting the $y=y k$ horizontal section, a finite number of points of the wear geometry, in which the $x$, $z$ coordinates respectively expressed as $\left(x_{1} k, z_{1} k\right),\left(x_{2} k, z_{2} k\right)$, $\ldots,(x n k, z n k)$, can be determined. Thus, the wear geometry function $z k=z(x k)$ can be constructed by the piecewise linear interpolation function. The Delaunay triangular mesh generation algorithm [11] is adopted for solving (xik, zik), $(i=1$, $2, \ldots, n)$. Let us define a discrete data collection $\Omega=(x i j, y i j)(i=$ $1,2, \ldots, n ; j=1,2, \ldots, m)$. Then $\Omega$ can be split into $N$ triangular elements $e l(I=1,2, \ldots, N)$. For any triangular element $e l$, the three vertices are assumed counterclockwise as $\left(x_{1}, y_{1}\right),\left(x_{2}, y_{2}\right)$, $\left(x_{3}, y_{3}\right)$ and the values of the triangular element $e /$ are assumed

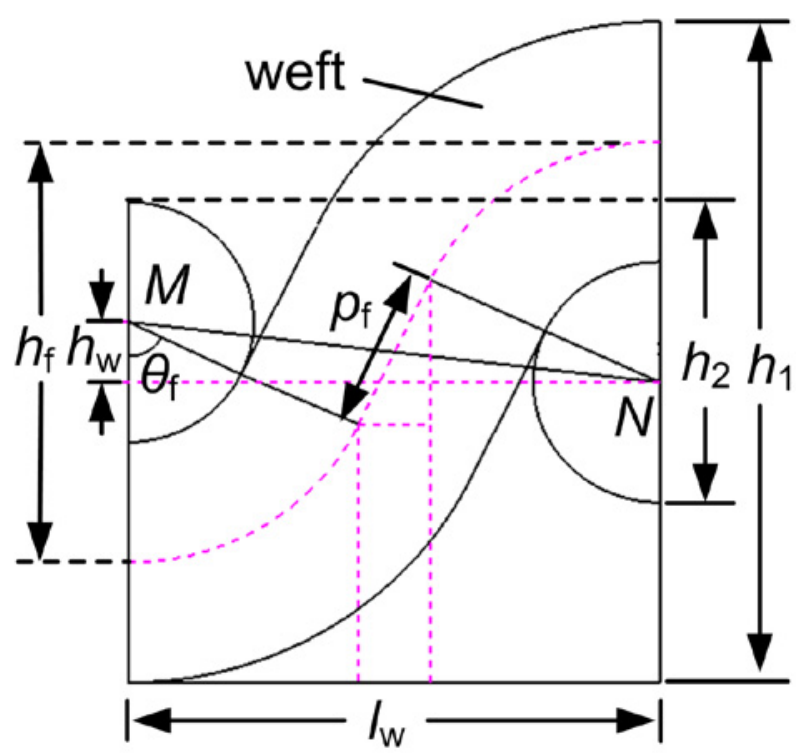

Figure 2. Geometrical description of weft yarn

as $z_{1}\left(x_{1}, y_{1}\right), z_{2}\left(x_{2}, y_{2}\right), z_{3}\left(x_{3}, y_{3}\right)$. Thus, the linear interpolation function $z(x, y)$ of the triangular element $e /$ can be expressed as

$z(x, y)=N_{1}(x, y) z_{1}+N_{2}(x, y) z_{2}+N_{3}(x, y) z_{3}$

where $N_{1}(x, y), N_{2}(x, y), N_{3}(x, y)$ are the linear interpolation basis functions,

$N_{1}(x, y)=\left(a_{1} x+b_{1} y+c_{1}\right) / 2 \Delta, \quad N_{2}(x, \quad y)=\left(a_{2} x+b_{2} y+c_{2}\right) / 2 \Delta, \quad N_{3}(x$, $y)=\left(a_{3} x+b_{3} y+c_{3}\right) / 2 \Delta$

where $\Delta$ is the area of the triangular element $e l$, , $\Delta=\frac{1}{2}\left|\begin{array}{ccc}x_{1} & y_{1} & 1 \\ x_{2} & y_{2} & 1 \\ x_{3} & y_{3} & 1\end{array}\right|, \begin{aligned} & a_{1}=y_{2}-y_{3}, a_{2}=y_{3}-y_{1}, a_{3}=y_{1}-y_{2}, \\ & b_{1}=x_{3}-x_{2}, b_{2}=x_{1}-x_{3}, b_{3}=x_{2}-x_{1}, \\ & c_{1}=x_{2} y_{3}-y_{2} x_{3}, c_{2}=x_{3} y_{1}-y_{3} x_{1}, c_{3}=x_{1} y_{2}-y_{1} x_{2} .\end{aligned}$

Let us define that the wear geometry function $z k(x)$ is linear in each interval $\left[x i k, x\left(i+{ }_{1}\right) k\right]$ in the $y=y k$ horizontal section, thus, $z_{k}(x)$ can be expressed by the following piecewise linear interpolation function:

$$
z_{k}(x)=z_{i k}+\frac{z_{(i+1) k}-z_{i k}}{x_{(i+1) k}-x_{i k}}\left(x-x_{i k}\right)\left(x_{i k} \leq x \leq x_{(i+1) k} ; i=1,2, L, n-1\right)
$$

The area of a quarter unit cell of weft yarn in the $y=y k$ horizontal section can be expressed as

$$
S_{k}=\sum_{i=1}^{n-1} \int_{x_{i k}}^{x_{(i+1) k}} z_{k}(x) d x
$$

The area ratio of the weft yarn in the $y=y k$ horizontal section is expressed as $A_{\mathrm{f}}=2 S \mathrm{~K} /\left(I_{\mathrm{w}} I_{\mathrm{f}}\right)$

The area ratio $A_{w}$ of warp yarn in the $y=y_{k}$ horizontal section can be obtained by the aforementioned method as weft yarn. If the gap between warp yarn and weft yarn is filled with matrix resin, then the area ratio of matrix resin $A_{r}$ in the $y=y k$ horizontal section is $A_{\mathrm{r}}=1-A_{\mathrm{f}}-A_{\mathrm{w}}$. 
A

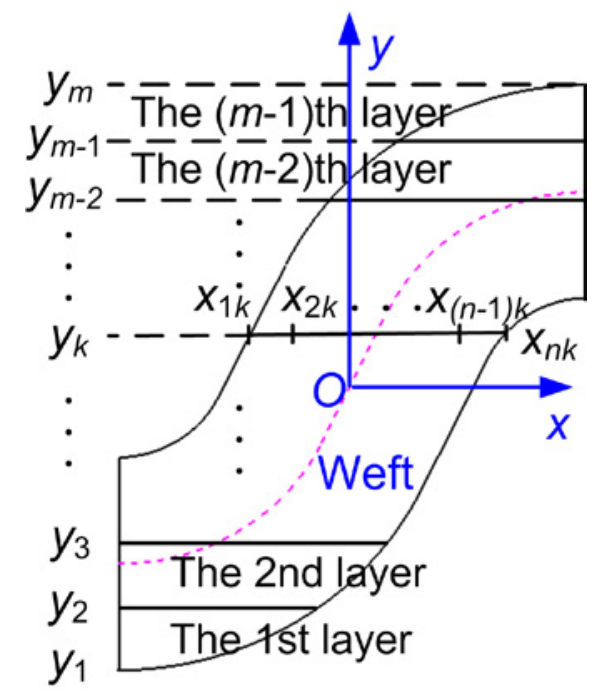

B

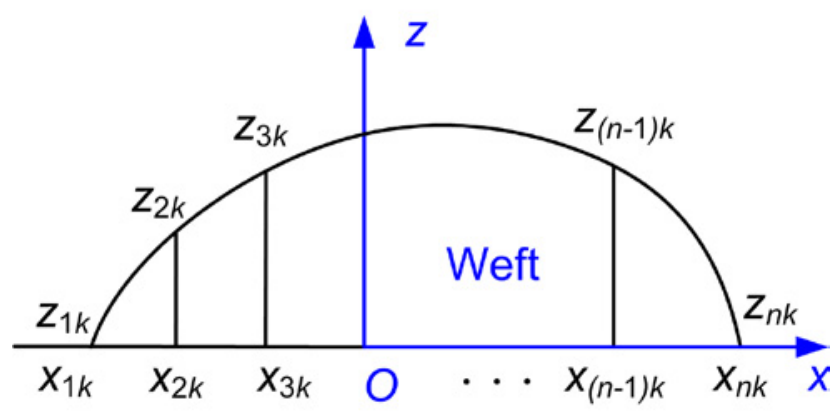

Figure 4. The stratified method used in a quarter unit cell of weft yarn. a) Sketch showing the stratified method $(z=0) \quad b)$ Sketch showing the wear geometry $(y=y k)$

\section{Results}

Figure $5 a$ and $b$ shows the three-dimensional simulation and the wear geometry variation of plain woven fabric, respectively, calculated in the case of the wear geometry model by Matlab software. As shown in Figure 5b, the wear geometries of weft yarns, warp yarns and the gaps (actually equal to the matrix resin for fabric composite) among the weft yarns and warp yarns change along with the thickness of fabric. Thus, as friction materials, the component area ratios of fabric composite keep changing with the wear depth. The component area ratios on the worn surface proved to be an useful instrument for assessing the wear resistance performance [8]. Thus, the area ratios of the wear geometries on each horizontal section of weft yarn, warp yarn and matrix resin are calculated by Matlab software.
Figure 6 and Figure 7 show, respectively, the component area ratio variations along with fabric composites thickness under different weft yarn diameters and different warp yarn diameters. As seen in Figure 6a) and Figure 7a), the thickness of the fabric composite increases with the increasing of weft and warp yarn diameters under the given condition. The area ratios of weft yarn and warp yarn increase respectively with the increasing of weft yarn diameter (Figure 6a) ) and warp yarn diameter (Figure 7b) ). As $h_{w}=0 \mathrm{~mm}$, the fabric is in the range of the first SP. So, in Figure $6 \mathrm{~b}$, the area ratio variations of warp yarn along with fabric composite thickness are almost identical under different weft yarn diameters, while the matrix resin area ratio decreases with the increasing of weft yarn diameters (Figure 6c) and warp yarn diameters (Figure 7c) ). The area ratios of weft yarn decrease with the increasing of warp yarn diameters in a specific range

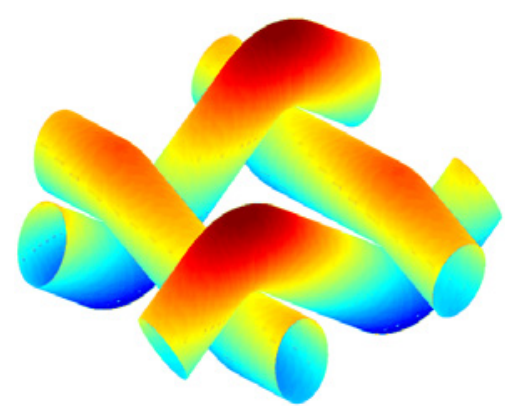

a) three-dimensional simulation

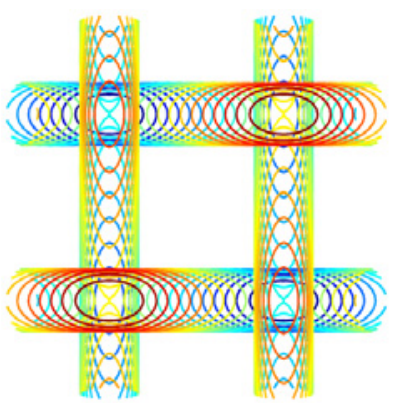

b) the wear geometry variation

Figure 5. An example of plain woven fabric based on the wear geometry model
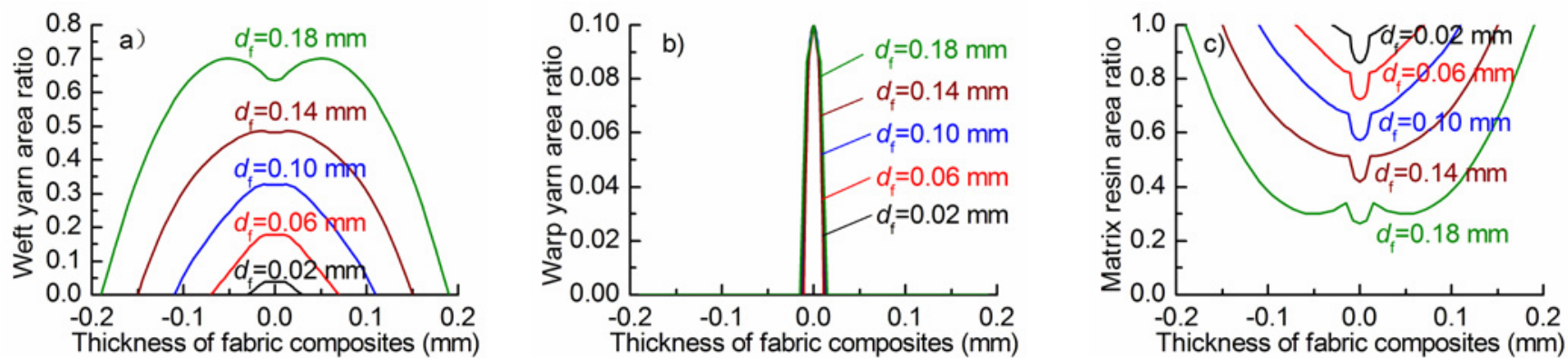

Figure 6. The component area ratio distribution under different weft yarn diameters $\left(l_{\mathrm{f}}=I_{\mathrm{w}}=0.2 \mathrm{~mm}, h_{\mathrm{w}}=0 \mathrm{~mm}\right.$ (the 1 st fabric structure phase), $d_{\mathrm{w}}=0.02$ $\mathrm{mm}$ ) 

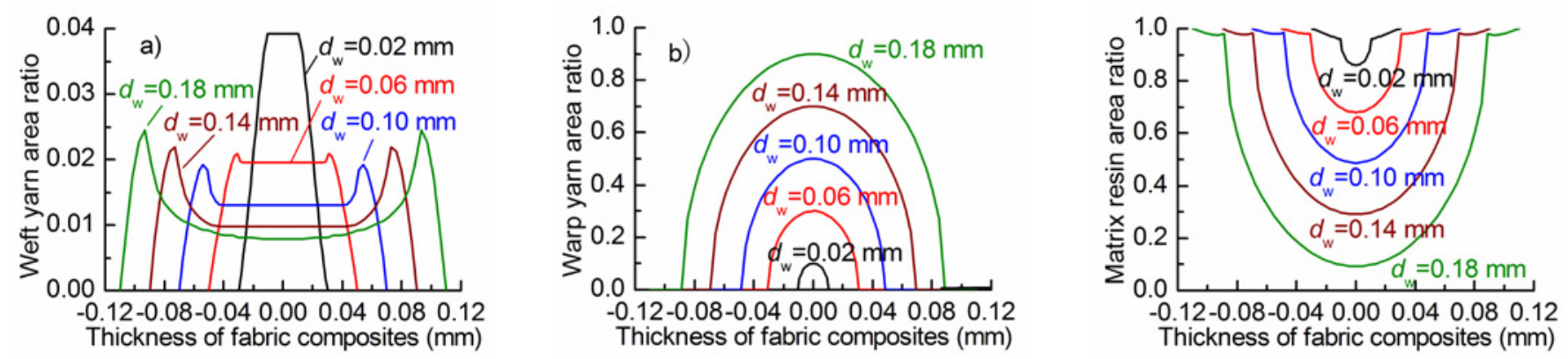

Figure 7. The component area ratio distribution under different warp yarn diameters $\left(l_{\mathrm{f}}=l_{\mathrm{w}}=0.2 \mathrm{~mm}, h_{\mathrm{w}}=0 \mathrm{~mm}\right.$ (the $1 \mathrm{st}$ fabric structure phase), $d_{\mathrm{f}}=0.02 \mathrm{~mm}$ )

of thickness of the fabric composite (Figure 7a); however, even if $d_{\mathrm{w}}=0.02 \mathrm{~mm}$, the maximum area ratio of weft yarn does not exceed 0.04 , which is very small in proportion relative to the warp yarn and matrix resin.

Figures 8 and 9 show, respectively, the component area ratio variations along with fabric composites thickness under different yarn-to-yarn distances of two adjacent weft yarns and two adjacent warp yarns. The changes in weft yarn-to-yarn and warp yarn-to-yarn distances have no effect on the thickness of fabric composites. As seen in Figure 8a, the changes in weft yarn-to-yarn distances have little influence on weft yarn area ratio variations. With increasing weft yarn-to-yarn distances, warp yarn area ratios decrease (Figure $8 b$ ) and the matrix resin area ratios increase (Figure $8 c$ ). The changes in warp yarn-to-yarn distances have no impact on warp yarn area ratio variations (Figure 9b). With the increasing of warp yarn-to-yarn distances, weft yarn area ratios decrease (Figure 9a) and the matrix resin area ratios increase (Figure $9 \mathrm{c}$ ).
Figure 10 shows the component area ratio variations along with fabric composites thickness under different fabric SPs, namely, the first $\left(h_{w}=0 \mathrm{~mm}\right)$, the third $\left(h_{w}=0.04 \mathrm{~mm}\right)$, the fifth $\left(h_{\mathrm{w}}=0.08 \mathrm{~mm}\right)$, the seventh $\left(h_{\mathrm{w}}=0.12 \mathrm{~mm}\right)$ and the ninth $\left(h_{\mathrm{w}}=0.16 \mathrm{~mm}\right) \mathrm{SPs}$. The thickness of fabric composites first decreases from the first SP to the fifth SP, then increases from the fifth SP to the ninth SP. The fabric SPs have obvious influence on the component area ratio variations. However, when the weft diameter is equal to warp diameter and weft yarn-to-yarn distance is equal to warp yarn-to-yarn distance, weft yarn area ratio variations under the first, the third, the fifth, the seventh, the ninth SPs are, respectively, are precisely the same as warp yarn area ratio variations under the ninth, the seventh, the fifth, the third, the first SPs(Figure $10 \mathrm{a}$ and $\mathrm{b}$ ). This phenomenon coincides with the conventional understanding, verifying the validity of such a model. Thus, the matrix resin area ratio variations under the first and the third SPs are, respectively, the same as the ninth and the seventh SPs.
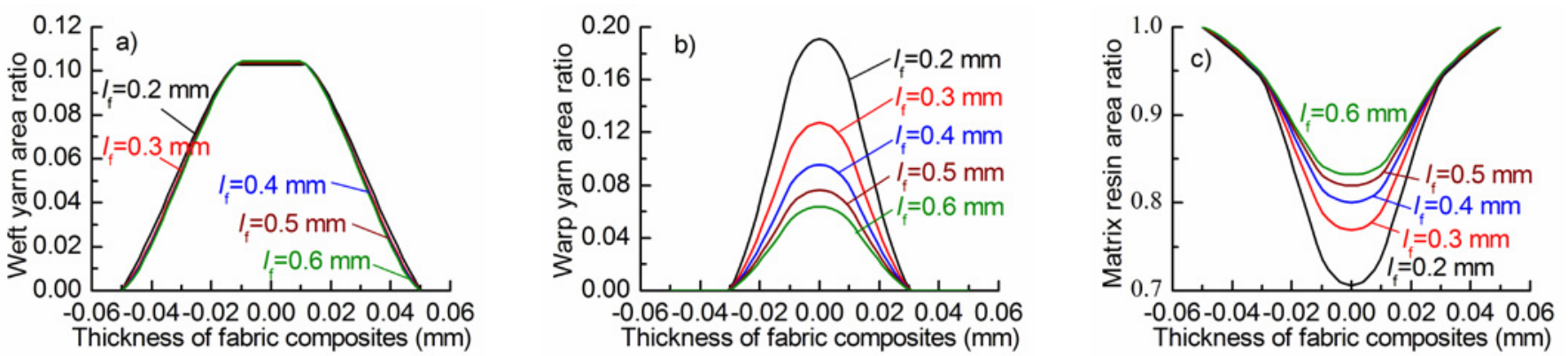

Figure 8. The component area ratio distribution under different weft yarn-to-yarn distances $\left(d_{\mathrm{f}}=d_{\mathrm{w}}=0.04 \mathrm{~mm}, h_{\mathrm{w}}=0.04 \mathrm{~mm}\right.$ (the $3 \mathrm{rd}$ fabric structure phase), $I_{\mathrm{w}}=0.2 \mathrm{~mm}$ )
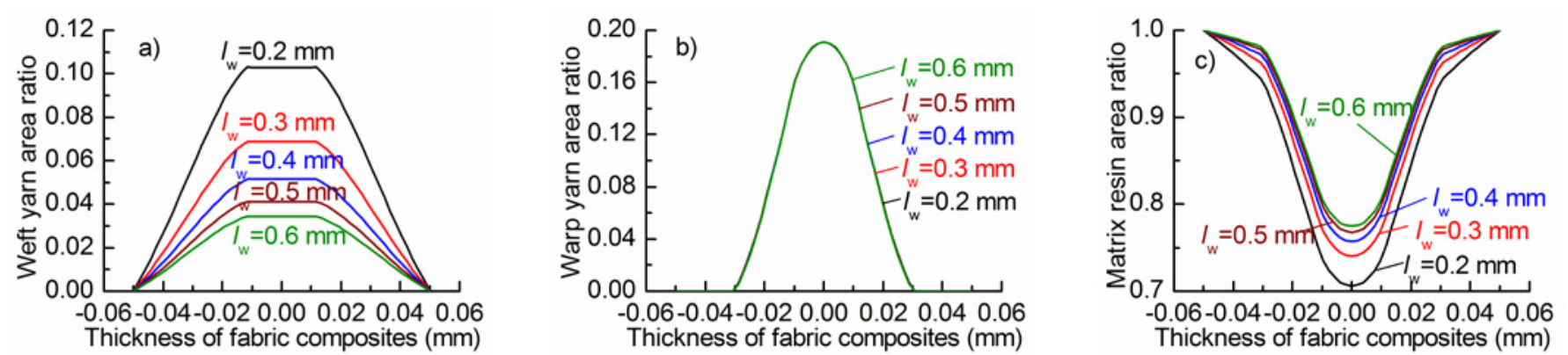

Figure 9. The component area ratio distribution under different warp yarn-to-yarn distances $\left(d_{\mathrm{f}}=d_{\mathrm{w}}=0.04 \mathrm{~mm}, h_{\mathrm{w}}=0.04 \mathrm{~mm}\right.$ (the $3 \mathrm{rd}$ fabric structure phase), $\left.l_{\mathrm{f}}=0.2 \mathrm{~mm}\right)$ 

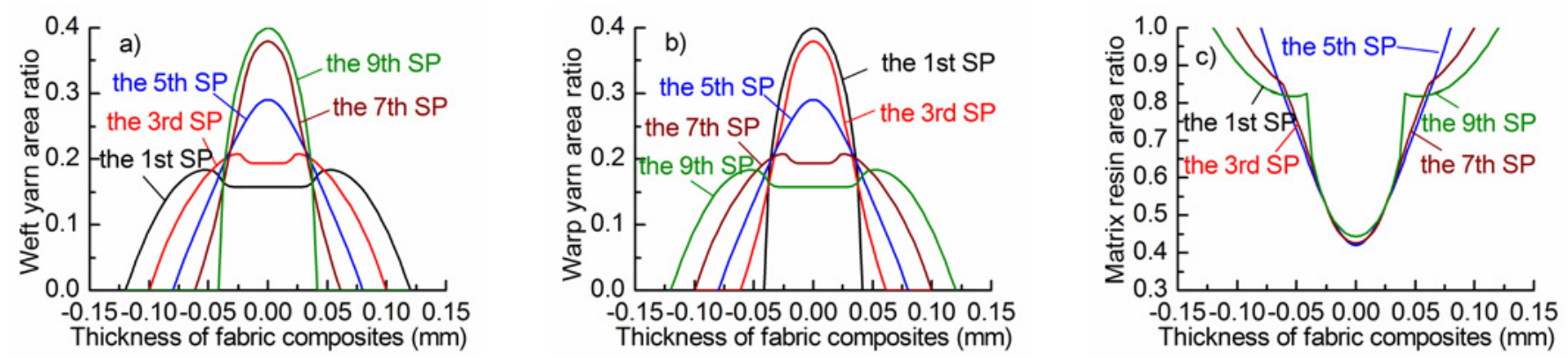

Figure 10. The component area ratio distribution under different fabric structure phases $\left(d_{\mathrm{f}}=d_{\mathrm{w}}=0.08 \mathrm{~mm}, I_{\mathrm{w}}=l_{\mathrm{f}}=0.2 \mathrm{~mm}, h_{\mathrm{w}}=0,0.04,0.08,0.12\right.$, $0.16 \mathrm{~mm}$ )

\section{Wear Experiment}

A wear experiment of PTFE/Kevlar fabrics has been performed on the MMU-5G. As shown in Figure 11, this test method utilized a ring-on-plate friction and wear form. A load of 2000 $\mathrm{N}$ was used. The rig stopped after specified rotations, and the wear depth and wear surface of each sample were recorded and observed. Three samples were repeated. The measured diameters of PTFE and Kevlar yarns are, respectively, 0.090 $\mathrm{mm}$ and $0.140 \mathrm{~mm}$. The measured yarn-to-yarn distances of two adjacent PTFE and Kevlar are, respectively, $0.368 \mathrm{~mm}$ and $0.541 \mathrm{~mm}$. The flexion heights of PTFE is $0.305 \mathrm{~mm}$. Measured and theoretical values of Kevlar area ratio are shown in Figure 11. Within a certain thickness of fabric, the trend of theoretical analysis of the Kevlar area ratio variations is in agreement with the measured results. The result shows that the proposed model is reasonable and effective in the variations of trend; however, an inherent large error exists in that the geometry of this model is different from the real geometry of the cross section of fabric. Thus, further research is needed to consider the real geometry of fabric. The wear performance experiment results show that the wear depth of the first test of each sample is larger than that of others, though the rotations (900 rotations) of the first test are far fewer than that of others $(2100,6000$, 6000 and 6000 rotations). This is because the height of the PTFE is greater than the height of Kevlar, and as is well known, the wear resistance performance of PTFE is worse than that of the Kevlar. So the wear depth is large at the beginning of the wear experiment for more PTFE on the surface of PTFE/Kevlar fabric. When the area ratio of Kevlar on the fabric worn surface is large, the wear resistance performance improves and the variations of wear depth are smaller.

\section{Conclusions}

Woven fabric composites are used as friction materials in many diverse fields. Due to the textile structure, the component distribution of such material is uneven. In other words, the component wear geometry or the area ratio changes with the wear depth. Thus, the wear resistance performance varies with the wear depth [8]. A wear geometry model of plain woven fabric composites is presented with the aim to clear the component area ratio variations along with the thickness of fabric composites under different textile structures. The computational data obtained from the wear geometry model

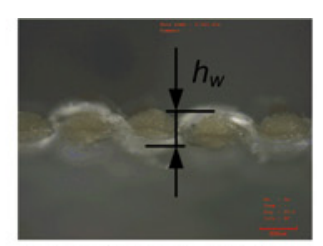

Cross-section of fabric

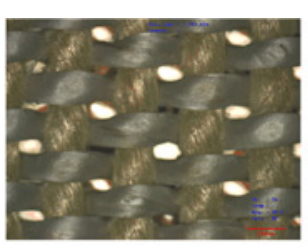

Before wear

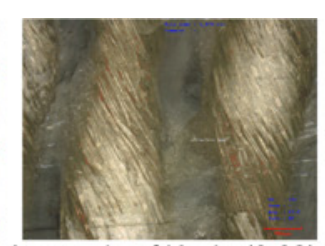

Area ratio of Kevlar(0.62)

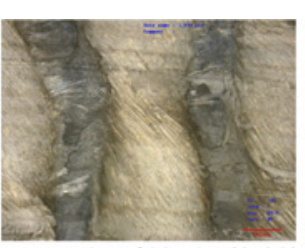

Area
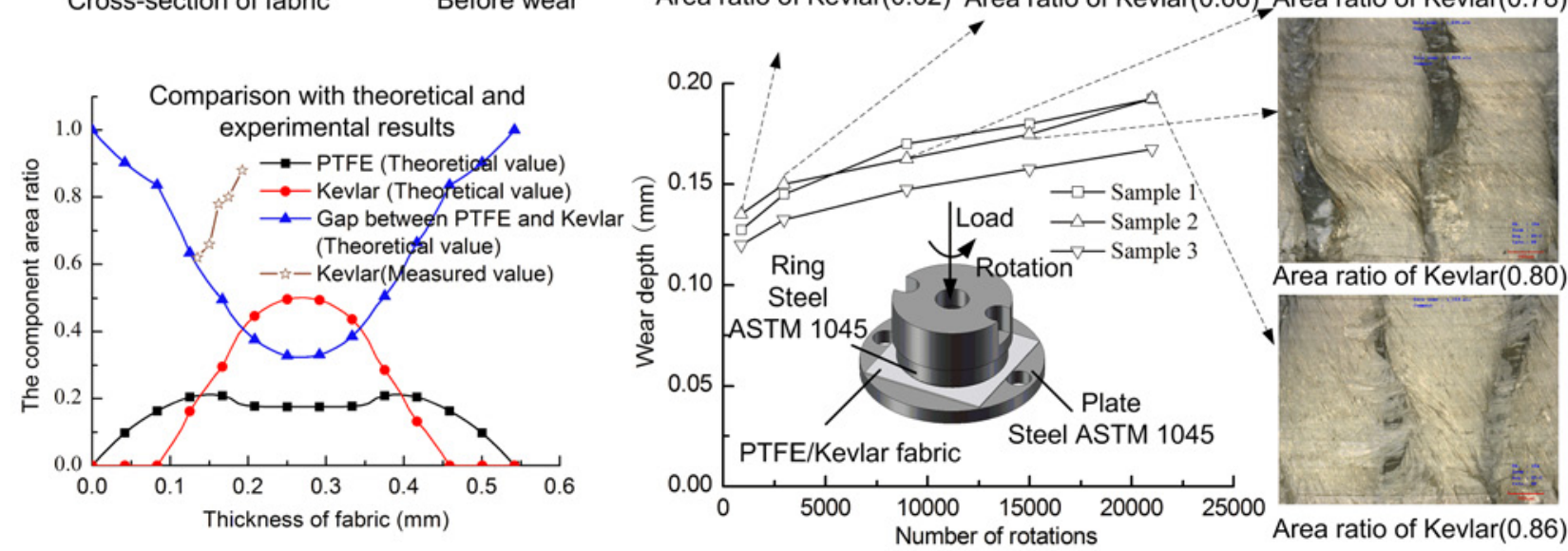

Figure 11. Schematic of pin-on-flat configuration and measured and theoretical value of weft yarn (PTFE) area ratio 
under five selected parameters, namely, weft and wrap yarn diameters, weft and wrap yarn-to-yarn distances and fabric SPs, were compared, respectively. By comparison of theoretical and experimental results, it can be concluded that the model has an inherent large error as it is highly idealised from the real fabric. However, it can reveal a trend of the component area ratio variations along with the thickness of fabric composites, and can be used as the basis of the follow-up model which is close to the actual fabric. It is also valuable for estimation of the wear resistance performance with different textile structures by the component wear geometry variations.

\section{Acknowledegment}

This work was supported by National Natural Science Foundation of China (Grant No.51405422), National Science and Technology Support Project (Grant No. 2014BAF08B03), Science and Technology Support Project of Qinhuangdao (Grant No. 201401A005), Young Teacher Autonomy Subject of Yanshan University (Grant No. 13LGB001).

\section{References}

[1] Huang T., Li T., Xin S., et al.: Mechanical and tribological properties of hybrid fabric-modified polyetherimide composites, Wear, Vol.306(1-2), pp. 64-72, 2013.

[2] Sharma M., Bijwe J., Mitschang P.: Wear performance of $P E E K$-carbon fabric composites with strengthened fibermatrix interface, Wear, Vol. 271(9-10), pp. 2261-2268, 2011.
[3] Ren G., Zhang Z., Zhu X., et al.: Influence of functional graphene as filler on the tribological behaviors of Nomex fabric/phenolic composite, Composites: Part A, Vol. 49, pp. 157-164, 2013.

[4] Bijwe J., Rattan R.: Influence of weave of carbon fabric in polyetherimide composites in various wear situations, Wear, Vol. 263(7-12), pp. 984-991, 2007.

[5] Sharma M., Rattan R., Batra N.K. Characteristics of Polyetherimide with carbon fabric composites in adhesive wear, International Journal of Current Engineering and Technology, Vol. 3(5), pp. 1992-1995, 2013.

[6] Gu D., Yang Y., Qi X., et al.: Influence of weave structures on the tribological properties of hybrid Kevlar/PTFE fabric composites, Chinese Journal of Mechanical Engineering, Vol. 25(5), pp. 1044-1051, 2012.

[7] Sharma M., Tiwari S., Bijwe J.: Optimization of material parameters for development of polyetherimide composites, Materials Science and Engineering B, Vol.168, pp. 55-59, 2010.

[8] Koltysheva N.G., Lomov S.V., Truevtzev N.N.: Abrasion resistance of Cotton/Flax fabrics: $3 D$ computer simulations of fabric wear geometry, AUTEX Research Journal, Vol. 4(4), pp. 182-186, 2004.

[9] Peirce F T., The Geometry of cloth structure, Journal of the Textile Institute Transactions, Vol. 46(5), pp. 45-96, 1937.

[10] Yang Y., Gu D., QI X., et al. A new elliptical cross-section mathematical model of woven fabric based on Jacobian transformation. Journal of Donghua University (English Edition), Vol. 29(5), pp. 434-438, 2012.

[11] Jonathan R. S.: Reprint of: Delaunay refinement algorithms for triangular mesh generation, Computational Geometry, Vol. 47(7), pp. 741-778, 2014. 